\title{
Long-term effect of anticoagulation following left atrial appendage occlusion with the LARIAT device in patients with nonvalvular atrial fibrillation: impact on thromboembolism, bleeding and mortality. Real life data
}

\author{
Radosław Litwinowicz ${ }^{1}$, Grzegorz Filip ${ }^{1}$, Dorota Sobczyk ${ }^{2}$, Dhanunjaya Lakkireddy ${ }^{3}$, \\ Venkat L.K. Vuddanda ${ }^{4}$, Magdalena Bartuśs ${ }^{5}$, Bogusław Kapelak ${ }^{1}$, Krzysztof Bartuś ${ }^{1}$ \\ ${ }^{1}$ Department of Cardiovascular Surgery and Transplantology, Institute of Cardiology, Jagiellonian University Medical College, Krakow, Poland \\ Emergency and Admission Department, John Paul II Hospital, Krakow, Poland \\ ${ }^{3}$ The Kansas City Heart Rhythm Institute, Overland Park Regional Hospital, University of Kansas, Kansas, USA \\ ${ }^{4}$ Brigham and Women's Hospital, Harvard Medical School, Boston MA, USA \\ ${ }^{5}$ Department of Pharmacology, Jagiellonian University Medical College, Krakow, Poland
}

Adv Interv Cardiol 2020; 16, 1 (59): 89-96

DOI: https://doi.org/10.5114/aic.2020.93916

\begin{abstract}
A bstract
Introduction: Indications for left atrial appendage occlusion (LAAO) are varied and patients require individual management strategies. However, currently no guidelines exist for postprocedure oral anticoagulation (OAC) after an LAAO procedure.

Aim: To evaluate the effect of OAC on thromboembolism, bleeding and mortality following the LAAO procedure for patients with AF.

Material and methods: One hundred and thirty-nine consecutive patients with nonvalvular atrial fibrillation (NVAF) who underwent LAAO with the LARIAT device were followed for at least 40 months. Decisions about OAC and its modifications were individualized based on clinical presentation, patient and physician preferences.

Results: Following LAAO, 52 (41\%) patients did not receive OAC (No-OAC group) and 75 (59\%) patients received OAC (OAC group), without any intergroup differences in $\mathrm{CHADS}_{2}, \mathrm{CHA}_{2} \mathrm{DS}_{2}-\mathrm{VASc}$ score or other thromboembolic risk factors. The median HAS-BLED score was higher in the OAC-group (median 3 (3-4) vs. 3 (2-4), $p=0.014$ ). During a median follow-up time of 51 (43-57) vs. $55(48-59)$ months in the No-OAC group and in the OAC group $(p=0.19)$ there were no significant differences between groups in ischemic stroke/TIA, $0(0 \%)$ vs. $2(2.7 \%)$, other thromboembolic events, $0(0 \%)$ vs. $1(1.3 \%)$, life-threatening, disabling or major events, $2(3.7 \%)$ vs. 2 (2.7\%), or annual mortality rate, $1.9 \%$ vs. $0.9 \%$, respectively.

Conclusions: There is no need for OAC after the LAAO procedure. Omitting OAC after an LAAO procedure in AF patients: (1) has similar stroke prevention rates as patients on OAC, (2) has similar bleeding prevention rates as patients on OAC, (3) has similar safety endpoints and long-term efficacy as patients on OAC.
\end{abstract}

Key words: stroke, atrial fibrillation, left atrial appendage, left atrial appendage occlusion, anticoagulant treatment.

Su m m a ry

There is no need for oral anticoagulation after the left atrial appendage occlusion procedure. Discontinuation or oral anticoagulation (OAC) continuation has a similar effect on stroke rate. Discontinuation or OAC continuation has a similar effect on bleeding rate. Discontinuation or OAC continuation has similar safety endpoints and efficacy.

\section{Introduction}

Percutaneous left atrial appendage occlusion (LAAO) has emerged as an acceptable alternative treatment for patients with nonvalvular atrial fibrillation (NVAF) in whom oral anticoagulation (OAC) therapy is contraindicated or anticoagulation therapy is ineffective [1-4]. Con-

Corresponding author:

Radosław A. Litwinowicz MD, PhD, Department of Cardiovascular Surgery and Transplantology, Institute of Cardiology, Jagiellonian University Medical College, Pradnicka St, 31-2020 Krakow, Poland, phone: +48 1261430 75, e-mail: radek.litwinowicz@gmail.com

Received: 10.05.2019, accepted: 16.09.2019. 
sequently, the number of LAAO procedures has rapidly increased in recent years.

As the left atrial appendage is the source of thrombus in $90 \%$ of patients with $\operatorname{NVAF}[5,6]$ following LAAO, anticoagulation therapy may not be required. Currently, recommendations for postprocedure anticoagulation depend on the system used, varying from no treatment to life-long combination therapy with OAC and antiplatelet agents [1, 2, 7, 8]. Extended anticoagulation therapy should be considered when an endocardial system is employed (due to the thrombogenic nature of the occlusion device) [9], if the patient is at high risk of thromboembolic events or has experienced a stroke whilst on OAC. One further factor influencing the variability in prescribing practices may be that LAAO procedures are fairly new and may be relatively under-recognized by general internal medicine physicians.

Therefore, the optimal duration and the type of OAC treatment for patients with NVAF following LAAO remain unclear.

\section{Aim}

This study aims to address this problem by presenting the clinical outcomes of anticoagulation strategies in patients with NVAF after LAAO over more than 5.5 years of follow-up.

\section{Material and methods}

A prospective, single-center study was performed in 139 consecutive patients with NVAF who underwent LAAO with the LARIAT device (SentreHEART Inc, Redwood, CA) between December 2009 and December 2010. Inclusion and exclusion criteria for the LARIAT procedure have been described previously [10]. Patient data were collected for demographics, medical history, stroke risk (CHADS 2 and $\mathrm{CHA}_{2} \mathrm{DS}_{2}$-VAS scores), bleeding (HAS-BLED score), and LAA dimensions. The study was approved by the local Ethics Committee and the Polish Ministry of Health.

\section{Postprocedure anticoagulation}

Aspirin monotherapy was recommended as postprocedure antithrombotic therapy. However, the final treatment was individualized based on the patients' history, contraindications, risk of stroke and bleeding, and the preferences of both the patients and the treating physicians.

The cohort was divided into 2 groups. The No-OAC group patients received no anticoagulation or antiplatelet agents (aspirin or clopidogrel), whilst the OAC group received anticoagulation therapy with vitamin $\mathrm{K}$ antagonists (VKAs) (warfarin or acenocoumarol) or a new anticoagulant (NOAC) (dabigatran or rivaroxaban).

\section{Thromboembolism reduction calculation}

Similar to our previous study [11, 12], adverse events were reported during follow-up visits based on the Munich LAA consensus document [13]. We recorded cardiovascular, noncardiovascular, procedural and immediate procedural mortality, thromboembolic events (stroke, transient ischemic attack (TIA), systemic embolism), life-threatening, disabling or major bleeding events, including fatal bleeding; symptomatic bleeding in a critical organ, such as intracranial, intraspinal, intraocular, or intramuscular bleeding with compartment syndrome; bleeding causing hypovolemic shock or severe hypotension requiring vasopressors or surgery; overt source of bleeding with a drop in hemoglobin $\geq 5 \mathrm{~g} / \mathrm{dl}$ or whole blood or packed red blood cell (RBC) transfusions of $\geq 4$ units; and mortality. The efficacy of the procedure to prevent thromboembolic events (stroke, TIA, systemic embolism, or thrombus in the heart chamber) was calculated by comparing the actual event rate with the event rate predicted by the $\mathrm{CHA}_{2} \mathrm{DS}_{2}$-VASC scores [2, 3, 14]. The annual risk of individual patients was recorded, and the average annual risk for the entire study population was calculated. Thromboembolism reduction was calculated as follows: (estimated \% - actual \% event rate)/estimated \% event rate) [15].

Bleeding reduction was assessed using the same method as that used for stroke reduction. The total number of major bleeding events per year was compared with the number of events predicted by the HAS-BLED score [16]: (estimated \% - actual \% event rate)/estimated $\%$ event rate) [15].

\section{Statistical analysis}

Continuous variables were analyzed for normal distribution using the Shapiro-Wilk test. Data are expressed as the mean \pm standard deviation or median (interquartile range; $\mathrm{Q} 1-25^{\text {th }}$ percentile and $\mathrm{Q} 3-75^{\text {th }}$ percentile), unless otherwise stated. If a nonparametric test was used, the obtained data were additionally presented as the mean \pm standard deviation for a better comparison with other studies. To assess the differences between two continuous variables, Student's $t$-test (for normally distributed values) or the Mann-Whitney U-test (values not normally distributed) were used. Categorical variables were expressed as counts and percentages. Baseline characteristics between the groups were compared using the $t$-test for continuous variables and the $\chi^{2}$ test for categorical variables. Kaplan-Meier analysis was performed to estimate survival over time. Statistical analyses were performed with Statistica 10.0 (StatSoft, Tulsa, OK, USA). A two-sided $p$-value $<0.05$ was considered statistically significant.

\section{Results}

The LAAO procedure was performed in 139 patients. One year after the procedure, 12 patients were lost to 
follow-up. Based on the data obtained from the Ministry of Health, it was possible to determine the status of the patients who were lost to follow-up. At the date of the last follow-up visit, 10 patients were still alive, and 2 patients had died.

At the end of the follow-up period, there were 52 (41\%) patients in the No-OAC group and 75 (59\%) patients in the OAC group. Median follow-up was 51 (4357) months in the No-OAC group and 55 (48-59) months in the OAC group. The total follow-up duration for the study population was 532.7 patient-years. There were no differences in age, sex, comorbidities, LAA anatomy and dimensions, median follow-up, $\mathrm{CHADS}_{2}$ and $\mathrm{CHA}_{2} \mathrm{DS}_{2}$-VAS scores between the groups (Table I). The median HASBLED score was significantly lower in the No-OAC group than in the OAC group (3 (2-4) vs. $3(3-4)(p=0.014))$ (Table I). There were no significant differences in the preprocedure anticoagulation regimens: in the No-OAC group, $81 \%$ of patients received VKAs, $13 \%$ antiplatelet agents and $6 \%$ no anticoagulation; in the OAC group, $87 \%$ of patients received VKAs and $13 \%$ antiplatelet agents. There were significant differences in the indications for LAAO between the No-OAC and OAC groups: LAAO was performed in $8 \%$ vs. $28 \%$ of patients with a history of a prior stroke/TIA while receiving OAC; in $23 \%$ vs. $10 \%$ of patients because of failure/complication while receiving OAC (other than stroke/TIA); $27 \%$ vs. $6 \%$ of patients because of contraindications to OAC; $42 \%$ vs. $51 \%$ of patients because of labile international normalized ratio (INR) (all $p<0.001$ ), respectively.

During the follow-up period, at 9-19 months, $48 \%$ of patients received no OAC, 52\% received a VKA, and 0\% received a NOAC; at $36-48$ months, $41 \%$ of patients received no OAC, $49 \%$ received a VKA, and $10 \%$ received a NOAC; at $54-60$ months, $42 \%$ of patients received no OAC, $40 \%$ received a VKA, and $18 \%$ received a NOAC, with no significant differences (Figure 1). Forty-seven percent of patients did not receive any anticoagulation treatment or antiplatelet agents during a 1-year follow-up.

\section{Thromboembolic risk reduction}

There were no thromboembolic events in the No-OAC group. In the OAC group, thromboembolic events were observed in 3 patients ( $4 \%, p=0.39)$. There was one episode of ischemic stroke after 12 months in a 58-year-old patient who was taking warfarin $\left(\mathrm{CHA}_{2} \mathrm{DS}_{2}\right.$-VASc score $=$ 6 and a history of previous stroke), one episode of TIA after 28 months in a 56-year-old patient also taking warfarin $\left(\mathrm{CHA}_{2} \mathrm{DS}_{2}\right.$-VASc score $\left.=2\right)$, and one episode of arterial peripheral embolism after 44 months in a 73-year-old patient who was taking acenocoumarol ( $\mathrm{CHA}_{2} \mathrm{DS}_{2}$-VASC score $=2$ and with deep vein thrombosis). The estimated thromboembolic risk reductions in the No-OAC and OAC groups are shown in Figure 2.

\section{Bleeding risk reduction}

In the follow-up period, there were 4 severe bleeding events: two hemorrhagic strokes occurred in the NoOAC group; in the OAC group, there was one hemorrhagic stroke in a 61-year-old patient who was taking acenocoumarol, and one gastrointestinal bleed in a 64-yearold patient taking dabigatran $(p=0.89)$. The estimated bleeding risk reductions in the No-OAC and OAC groups are presented in Figure 3.

\section{Mortality rate}

The annual mortality rate was $1.9 \%$ in the No-OAC group (a total of 4 patients, including 2 cardiovascular deaths, 1 noncardiovascular death, and 1 death of unknown cause) and $0.93 \%$ in the OAC group (a total of 3 patients, 2 cardiovascular deaths, and 1 death of unknown cause). Kaplan-Meier survival analysis revealed no differences during the complete follow-up period (Figure 4).

\section{Discussion}

We present the first long-term outcome of oral anticoagulation therapy after a successful LAAO procedure. In our patients, postprocedure oral anticoagulation therapy (VKAs or NOACs) following the LAAO procedure with a LARIAT device did not reduce the risk of stroke, systemic thromboembolism, bleeding complications, or mortality during 5.5 years of follow-up.

Current ESC [2] and ACC/AHA/HRS [3] guidelines include a Class IA recommendation for oral anticoagulation in patients with atrial fibrillation (AF) with a history of a prior stroke or a $\mathrm{CHA}_{2} \mathrm{DS}_{2}$-VAS score $\geq 2$. However, for cases in which OAC is contraindicated or ineffective, the LAAO procedure represents a feasible alternative, even in elderly patients [11]. In the last decade, the LAAO procedure for the management of patients with AF has been growing in popularity [8]. The ESC and AHA guidelines give the LAAO procedure a Class $2 \mathrm{~B}$ recommendation for patients with atrial fibrillation in whom anticoagulation is contraindicated $[2,3]$. However, at present, there are no universal guidelines for initiating post-LAAO anticoagulation [1, 17]. Because heterogeneous patient groups result in treatment variations, an analysis of the requirements for and the safety of ongoing LAAO treatment is paramount $[1,2]$.

LAAO procedures utilizing endocardial devices such as the Watchman or the Amulet have the highest risk of thromboembolic events in the first 6 weeks after the procedure $[7,8]$. The LAA occlusion device may act as a thrombogenic focus in the absence of OAC or anticoagulation therapy in general [17]. Preclinical studies have shown complete epithelialization of the device surface by 45 days [18]. Because the prevention of acute device-related thrombosis is critical following the initial procedure, the PROTECT AF and PREVAIL trials used postprocedure treatment protocols of VKA anticoagulation therapy for 
Table I. Baseline clinical characteristics

\begin{tabular}{|c|c|c|c|}
\hline Parameter & No-OAC group $(n=52)$ & OAC group $(n=75)$ & $P$-value \\
\hline Age [years] & $60.7 \pm 10.2$ & $62 \pm 8.6$ & 0.21 \\
\hline Median follow-up [months] & $51(43-57)$ & $55(48-59)$ & 0.19 \\
\hline Sex (female) & $23(44 \%)$ & $35(47 \%)$ & 0.92 \\
\hline Median $\mathrm{CHADS}_{2}$ score: & $1(1-2.5)$ & $2(1-3)$ & 0.10 \\
\hline $\mathrm{CHADS}_{2}$ score 0 & $1(2 \%)$ & $2(3 \%)$ & \\
\hline $\mathrm{CHADS}_{2}$ score 1 & $29(56 \%)$ & $28(37 \%)$ & \\
\hline $\mathrm{CHADS}_{2}$ score 2 & $9(17 \%)$ & $25(33 \%)$ & \\
\hline $\mathrm{CHADS}_{2}$ score 3 & $12(23 \%)$ & $14(19 \%)$ & \\
\hline $\mathrm{CHADS}_{2}$ score 4 & $1(2 \%)$ & $6(8 \%)$ & \\
\hline $\mathrm{CHADS}_{2}$ score 5 & 0 & 0 & \\
\hline $\mathrm{CHADS}_{2}$ score 6 & 0 & 0 & \\
\hline Mean $\mathrm{CHADS}_{2}$ - score & $1.67 \pm 0.9$ & $1.9 \pm 1.0$ & - \\
\hline Median $\mathrm{CHA}_{2} \mathrm{DS}_{2}$-VASc score: & $2(1-4)$ & $3(2-4)$ & 0.74 \\
\hline $\mathrm{CHA}_{2} \mathrm{DS}_{2}$-VASc score 1 & $15(29 \%)$ & $14(19 \%)$ & \\
\hline $\mathrm{CHA}_{2} \mathrm{DS}_{2}$-VASc score 2 & $15(29 \%)$ & $20(27 \%)$ & \\
\hline $\mathrm{CHA}_{2} \mathrm{DS}_{2}$-VASc score 3 & $7(13 \%)$ & $12(16 \%)$ & \\
\hline $\mathrm{CHA}_{2} \mathrm{DS}_{2}$-VASc score 4 & $6(12 \%)$ & $12(16 \%)$ & \\
\hline $\mathrm{CHA}_{2} \mathrm{DS}_{2}$-VASc score 5 & $6(12 \%)$ & $13(17 \%)$ & \\
\hline $\mathrm{CHA}_{2} \mathrm{DS}_{2}$-VASc score 6 & $3(6 \%)$ & $3(4 \%)$ & \\
\hline $\mathrm{CHA}_{2} \mathrm{DS}_{2}$-VASc score 7 & 0 & $1(1 \%)$ & \\
\hline $\mathrm{CHA}_{2} \mathrm{DS}_{2}$-VASc score 8 & 0 & $1(1 \%)$ & \\
\hline $\mathrm{CHA}_{2} \mathrm{DS}_{2}$-VASc score 9 & 0 & 0 & \\
\hline Mean $\mathrm{CHA}_{2} \mathrm{DS}_{2}$-VASc score & $2.6 \pm 1.6$ & $3.1 \pm 1.7$ & - \\
\hline Median HAS-BLED score: & $3(2-4)$ & $3(3-4)$ & 0.014 \\
\hline HAS-BLED score 1 & $8(15 \%)$ & $2(3 \%)$ & \\
\hline HAS-BLED score 2 & $12(23 \%)$ & $12(16 \%)$ & \\
\hline HAS-BLED score 3 & $17(33 \%)$ & $32(43 \%)$ & \\
\hline HAS-BLED score 4 & $9(17 \%)$ & $20(27 \%)$ & \\
\hline HAS-BLED score 5 & $3(6 \%)$ & $6(8 \%)$ & \\
\hline HAS-BLED score 6 & $3(6 \%)$ & $3(4 \%)$ & \\
\hline Mean HAS-BLED score & $2.9 \pm 1.3$ & $3.3 \pm 1.0$ & - \\
\hline Previous CVA/stroke & $12(23 \%)$ & $19(25 \%)$ & 0.90 \\
\hline Hypertension & $47(90 \%)$ & $72(96 \%)$ & 0.36 \\
\hline Coronary artery disease & $8(15 \%)$ & $15(20 \%)$ & 0.42 \\
\hline DM 2 & $6(12 \%)$ & $19(25 \%)$ & 0.09 \\
\hline $\mathrm{COPD}^{\star *}$ & $2(4 \%)$ & $7(9 \%)$ & 0.41 \\
\hline Pacemaker/ICD & $7(13 \%)$ & $14(19 \%)$ & 0.37 \\
\hline AF ablation therapy & $7(13 \%)$ & $6(8 \%)$ & 0.48 \\
\hline \multicolumn{4}{|l|}{ LAA dimensions on CT: } \\
\hline Width & $30(23-33)$ & $30(22-32)$ & 0.42 \\
\hline Length & $30(25-40)$ & $30(25-36)$ & 0.78 \\
\hline Number of lobes & $1(1-2)$ & $2(1-2)$ & 0.44 \\
\hline \multicolumn{4}{|l|}{ Pre-procedure medication: } \\
\hline None & $3(6 \%)$ & 0 & 0.10 \\
\hline Antiplatelet agents & $7(13 \%)$ & $10(13 \%)$ & \\
\hline VKA & $42(81 \%)$ & $65(87 \%)$ & \\
\hline \multicolumn{4}{|l|}{ Indication for LAAC: } \\
\hline Stroke/TIA while on OAC & $4(8 \%)$ & $21(28 \%)$ & 0.001 \\
\hline Failure/complication* while on OAC & $12(23 \%)$ & $10(10 \%)$ & \\
\hline Contraindicated to OAC & $6(27 \%)$ & $6(6 \%)$ & \\
\hline Labile INR & $22(42 \%)$ & $38(51 \%)$ & \\
\hline
\end{tabular}

*Failure of OAC: history of left atrial/left atrial appendage thrombus despite OAC; complication of OAC: history of bleeding complication with OAC. 




Figure 1. Change in anticoagulation treatment after LAAO procedure over 5-year follow-up $(p<0.001)$

45 days, dual antiplatelet therapy for 6 months and aspirin thereafter $[19,20]$.

The LARIAT procedure uses a percutaneous approach to achieve suture closure of the LAA [12, 21-23]. In contrast to an endocardial system, epicardial LAAO exclusion with the LARIAT device leaves no foreign body, thereby minimizing the risk of postprocedure thrombus formation and a pericardial inflammatory response [18, 24]. However, histologic and anatomic analysis of the LAA after the LARIAT procedure suggests that a significant inflammatory response results in scarring and permanent closure of the LAA [22]. Therefore, our recommendation of life-long aspirin monotherapy differs from the standard protocols used following endocardial procedures

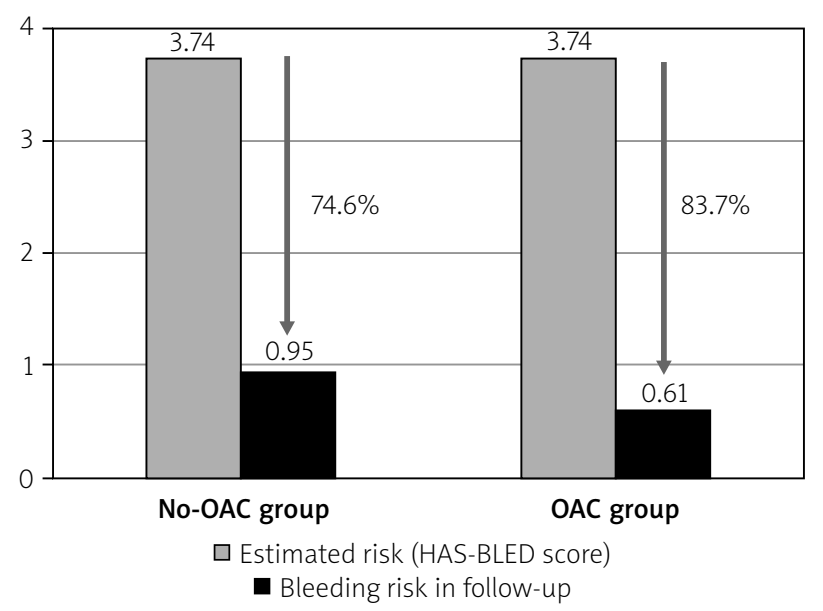

Figure 3. Estimated bleeding risk reductions in the No-OAC and OAC groups



Figure 2. Effectiveness in stroke risk reduction (100 patient-years) in the No-OAC group and OAC group during total follow-up

[19, 20, 25]. Sievert et al. [26] demonstrated that patients with contraindications to OAC therapy undergoing LAAO required no postprocedure anticoagulation regimen to prevent embolic events. The majority of these patients did not receive any postprocedure antithrombotic agents. Although we recommended this standard regimen for our patients, the presence of comorbidities and physician preference meant that some patients continued to receive OAC. We observed that the proportion of patients receiving OAC changed over time: before the procedure, $84 \%$ of patients received OAC, and only $16 \%$ received antiplatelet agents. Fifty-two percent of patients received OAC 1 year after the procedure, 59\% after 3 years, and $58 \%$ after 5 years. Notably, nearly $47 \%$ of patients received no anticoagulation treatment or antiplatelet agents during the follow-up.

There may be several reasons for the variations in anticoagulation therapy. The lack of ESC and AHA guide-

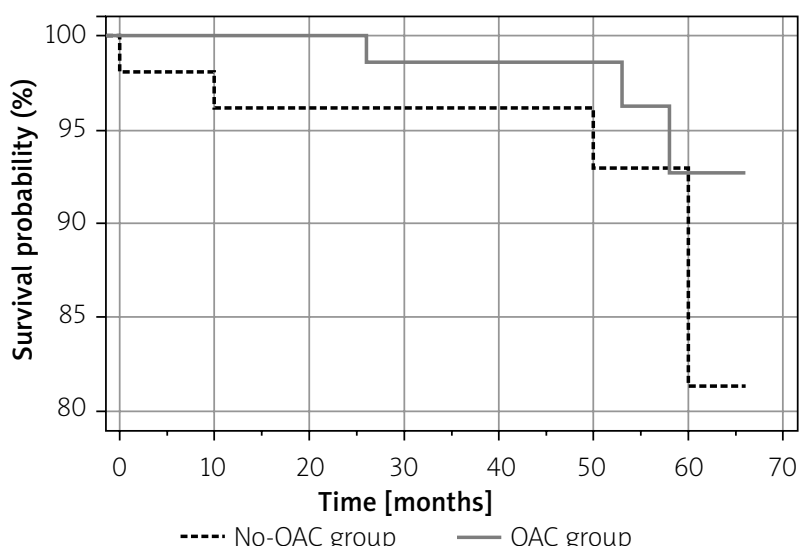

Figure 4. Kaplan-Meier survival analysis in the NoOAC group and OAC g during total follow-up (logrank test $p=0.28$ ) 
lines leaves physicians free to exercise their own discretion [2, 3]. Patient profiles also influence the choice; for instance, individual risks of thromboembolic events or hemorrhage should be taken into account. Special attention should also be paid to the patients with a history of a prior stroke, who had no contraindications to anticoagulation therapy and in whom left atrial appendage closure (LAAC) was performed because of the occurrence of a stroke/TIA while taking OAC. In contrast to ESC and AHA guidelines [2, 3], the Polish Cardiac Society expands LAAC indications to include patients with a history of cardioembolic stroke while taking OAC [1]. In our previous study, we demonstrated that patients with a history of a prior stroke may be the preferred group for LAAC, and LAAC may be preferred for all patients with a history of stroke/TIA regardless of the presence or absence of contraindications to anticoagulant therapy [27]. In the present study, continuation of anticoagulation therapy after LAAO was significantly higher in the subgroup of patients with a history of a previous stroke or TIA.

In addition, lack of familiarity with the relatively new LAAO procedure may make physicians more likely to continue OAC therapy. For example, we have observed situations in which physicians who have assumed the care of patients after an LAAO procedure insisted on using ongoing oral anticoagulation. Lastly, the addition of NOACs to the AF management guidelines partway through our follow-up period may have contributed to the observed rise in the number of patients receiving $\operatorname{OAC}[2,3]$.

In our study, the baseline thromboembolic risk was the same between the two groups (including $\mathrm{CHADS}_{2}$ score, $\mathrm{CHA}_{2} \mathrm{DS}_{2}$-VAS score plus sex, age, heart failure, hypertension or previous stroke/TIA). We observed no thromboembolic events in the No-OAC group. In the OAC group there was one episode of stroke (a patient with a $\mathrm{CHA}_{2} \mathrm{DS}_{2}$-VAS score of 6 with a history of previous stroke), one episode of TIA (a patient with a $\mathrm{CHA}_{2} \mathrm{DS}_{2}$ VAS score of 2), and one episode of arterial peripheral embolism (a patient with a $\mathrm{CHA}_{2} \mathrm{DS}_{2}$-VAS score of 2 and deep vein thrombosis). There was no significant difference between these results.

In our opinion, there are also several others factors, apart from AF, that increase the risk of thromboembolism and that may have contributed to these thromboembolic events. However, these factors are not included in the $\mathrm{CHA}_{2} \mathrm{DS}_{2}$-VAS score and include obesity, diet, smoking, dyslipidemia, physical activity, metabolic syndrome, carotid artery disease, alcohol consumption, sickle cell disease or, as in our patient, deep vein thrombosis [28]. We believe that postprocedure OAC therapy had no influence on the risk of thromboembolic events due to AF after LAA elimination.

Surprisingly, the median HAS-BLED score was significantly higher in the OAC group than in the No-OAC group, although the reduction in the risk for bleeding was similar in both groups. Preprocedure anticoagulation was similar between the groups, with more than $80 \%$ receiving VKAs. However, half of the patients in the No-OAC group had an LAAO procedure because of either a contraindication to anticoagulation therapy or failure/ complications resulting from anticoagulation treatments (50\% of patients in the No-OAC group vs. $16 \%$ of patients in the OAC group). Therefore, due to the higher risk of bleeding in the No-OAC group because of the preexisting complications of anticoagulant therapy, the frequency of bleeding between the groups was similar.

Of note, the addition of NOAC to the management protocols may have influenced the HAS-BLED scores. A favorable risk-benefit profile and a lower risk of major bleeding have led to NOACs increasingly replacing VKAs in the treatment of AF. Of course, NOACs are not free of complications [29]. Nonetheless, we observed no differences in the bleeding rate between the groups.

In our study, postprocedure LAAO anticoagulation treatment did not impact the mortality rate. Based on the large real-life registries from the Watchman and Amulet report, the highest risk of bleeding is observed within 6 weeks following an LAAO procedure, in cases in which OAC is used until device endothelialization is complete. However, mortality after LAAO is more likely due to patients' advanced age and considerable comorbidities rather than a result of bleeding complications [20, 21, 30].

Therefore, in our opinion, withholding oral anticoagulation treatment after the LAAO procedure with the LARIAT device is safe for patients after long-term follow-up.

This is a nonrandomized, retrospective, observational single-center study. The major limitations of our study include the estimation the overall value of LAAO, the lack of a control group, and the use of only a calculated stroke or bleeding risk score for analysis. In addition, the analyzed groups are small, and the results are underpowered. There were also no rigid criteria for postprocedure anticoagulation treatment, and the final decision was left to physician and patient preference. Lastly, we did not have information about whether the doses used in the postprocedure anticoagulation treatment were therapeutic. Because we analyzed only patients who underwent an LAAO procedure using the LARIAT, which is an epicardial device, our results cannot be easily extrapolated to patients undergoing other LAAO procedures.

\section{Conclusions}

Our long-term outcomes suggest that there is no need to administer oral anticoagulation after an LAAO procedure. When compared with patients receiving OAC, withholding OAC after an LAAO procedure in patients with $A F$ is associated with similar (1) stroke prevention rates, (2) bleeding prevention rates, and (3) safety endpoints and long-term efficacy. 


\section{Acknowledgments}

The authors would like to thank Professor Anetta Undas MD, PhD for her very valuable comments.

This study is the result of the research grant No. UMO-2014/13/D/NZ5/01351 funded by the National Science Centre.

\section{Conflict of interest}

Lakkireddy D. is the co-chair of the AMAZE trial steering committee and has received an institutional research grant. Bartus $\mathrm{K}$ is the recipient of a research grant. Other authors declare no conflict of interest.

\section{References}

1. Grygier M, Wojakowski W, Smolka G, et al. Left atrial appendage occlusion: consensus document of Association of Cardiovascular Interventions and Heart Rhythm Section of Polish Cardiac Society. Kardiol Pol 2018; 76: 677-97.

2. Kirchhof P, Benussi S, Kotecha D, et al. 2016 ESC Guidelines for the management of atrial fibrillation developed in collaboration with EACTS. Eur Heart J 2016; 37: 2893-962.

3. January CT, Wann LS, Calkins H, et al. 2019 AHA/ACC/HRS Focused Update of the 2014 AHA/ACC/HRS Guideline for the Management of Patients With Atrial Fibrillation: A Report of the American College of Cardiology/American Heart Association Task Force on Clinical Practice Guidelines and the Heart Rhythm Society. J Am Coll Cardiol 2019; 74: 104-32.

4. Burysz M, Litwinowicz R, Bryndza $M$, et al. Percutaneous left atrial appendage closure using the LAmbre device. First clinical results in Poland. Adv Interv Cardiol 2019; 15: 251-4.

5. Onalan O, Crystal E. Left atrial appendage exclusion for stroke prevention in patients with nonrheumatic atrial fibrillation. Stroke 2007; 38: 624-30.

6. Bartus K, Myc, J, Bartus M, et al. Rapid left atrial appendage thrombus formation in epicardial percutaneous LAA suture ligation with LARIAT. Adv Interv Cardiol 2018; 14: 435-7.

7. Price MJ. Left atrial appendage occlusion: data update. Interv Cardiol Clin 2018; 7: 159-68.

8. Baman JR, Mansour M, Heist EK, et al. Percutaneous left atrial appendage occlusion in the prevention of stroke in atrial fibrillation: a systematic review. Heart Fail Rev 2018; 23: 191-208.

9. Bartus K, Han FT, Bednarek J, et al. Percutaneous left atrial appendage suture ligation using the LARIAT device in patients with atrial fibrillation: initial clinical experience. J Am Coll Cardiol 2013; 62: 108-18.

10. Bartus K, Bednarek J, Myc J, et al. Feasibility of closed-chest ligation of the left atrial appendage in humans. Heart Rhythm 2011; 8: 188-93.

11. Litwinowicz R, Bartus M, Ceranowicz P, et al. Stroke risk reduction after left atrial appendage occlusion in elderly patients with atrial fibrillation: longterm results. Pol Arch Intern Med 2018; 128: 327-9.

12. Litwinowicz R, Bartus M, Ceranowicz P, et al. Left atrial appendage occlusion for stroke prevention in diabetes mellitus patients with atrial fibrillation: long term results. J Diabetes 2019; 11: 75-82.

13. Tzikas A, Holmes DR, Gafoor S, et al. Percutaneous left atrial appendage occlusion: the Munich consensus document on defi- nitions, endpoints, and data collection requirements for clinical studies. Europace 2017; 19: 4-15.

14. Melgaard L, Gorst-Rasmussen A, Lane DA, et al. Assessment of the CHA2DS2-VASC score in predicting ischemic stroke, thromboembolism, and death in patients with heart failure with and without atrial fibrillation. JAMA 2015; 314: 1030-8.

15. Freixa X, Gafoor S, Regueiro A, et al. Comparison of efficacy and safety of left atrial appendage occlusion in patients aged $<75$ to $\geq 75$ years. Am J Cardiol 2016; 117: 84-90.

16. Lip GY, Frison L, Halperin JL, et al. Comparative validation of a novel risk score for predicting bleeding risk in anticoagulated patients with atrial fibrillation: the HAS-BLED (Hypertension, Abnormal Renal/Liver Function, Stroke, Bleeding History or Predisposition, Labile INR, Elderly, Drugs/Alcohol Concomitantly) score. J Am Coll Cardiol 2011; 57: 173-80.

17. Pillarisetti J, Reddy YM, Gunda S, et al. Endocardial (Watchman) vs epicardial (Lariat) left atrial appendage exclusion devices: understanding the differences in the location and type of leaks and their clinical implications. Heart Rhythm 2015; 12: 1501-7.

18. Kar S, Hou D, Jones R, et al. Impact of Watchman and Amplatzer devices on left atrial appendage adjacent structures and healing response in a canine model. JACC Cardiovasc Interv 2014; 7: 801-9.

19. Holmes DR, Kar S, Price MJ, et al. Prospective randomized evaluation of the Watchman Left Atrial Appendage Closure device in patients with atrial fibrillation versus long-term warfarin therapy: the PREVAIL trial. J Am Coll Cardiol 2014; 64: 1-12.

20. Holmes DR, Reddy VY, Turi ZG, et al. Percutaneous closure of the left atrial appendage versus warfarin therapy for prevention of stroke in patients with atrial fibrillation: a randomised non-inferiority trial. Lancet 2009; 374: 534-42.

21. Bartus K, Podolec J, Lee RJ, et al. Atrial natriuretic peptide and brain natriuretic peptide changes after epicardial percutaneous left atrial appendage suture ligation using LARIAT device. J Physiol Pharmacol 2017; 68: 117-23.

22. Bartus K, Morelli RL, Szczepanski W, et al. Anatomic analysis of the left atrial appendage after closure with the LARIAT device. Circ Arrhythm Electrophysiol 2014; 74: 764-7.

23. Litwinowicz R, Bartus $M$, Burysz $M$, et al. Long term outcomes after left atrial appendage closure with the LARIAT device stroke risk reduction over five years follow-up. PLoS One 2018; 13: e0208710.

24. Bartuś K, Litwinowicz R, Dziewierz A, et al. Coronary artery bypass grafting after left atrial appendage ligation - is anti-inflammatory treatment after LARIAT effective? Adv Interv Cardiol 2018; 14: 438-9.

25. Litwinowicz R, Bartus M, Kapelak B, et al. Reduction in risk of stroke and bleeding after left atrial appendage closure with LARIAT device in patients with increased risk of stroke and bleeding: Long term results. Catheter Cardiovasc Interv 2019; 94: 837-42.

26. Sievert H, Rasekh A, Bartus K, et al. Left atrial appendage ligation in nonvalvular atrial fibrillation patients at high risk for embolic events with ineligibility for oral anticoagulation: initial report of clinical outcomes. JACC Clin Electrophysiol 2015; 1: 465-74.

27. Litwinowicz R, Bartus $M$, Malec-Litwinowicz $M$, et al. Left atrial appendage occlusion for secondary stroke prevention in patients with atrial fibrillation: long-term results. Cerebrovasc Dis 2019; 47: 188-95.

28. Kernan WN, Ovbiagele B, Black HR, et al. Guidelines for the prevention of stroke in patients with stroke and transient ischemic attack: a guideline for healthcare professionals from the Amer- 
ican Heart Association/American Stroke Association. Stroke 2014; 45: 2160-236.

29. Litwinowicz R, Konstanty-Kalandyk J, Goralczyk T, et al. Dabigatran level monitoring prior to idarucizumab administration in patients requiring emergent cardiac surgery. J Thromb Thrombolysis 2018; 45: 9-12.

30. Boersma LV, Ince H, Kische S, et al. Efficacy and safety of left atrial appendage closure with WATCHMAN in patients with or without contraindication to oral anticoagulation: 1-year follow-up outcome data of the EWOLUTION trial. Heart Rhythm 2017; 14: 1302-8. 